\title{
On the Feasibility of SISO/MIMO-PLC based on the Discrete Cosine Transform Type-III even
}

\author{
María Elena Domínguez-Jiménez, Gabriela Sansigre-Vidal, David Osés, and David Luengo \\ Universidad Politécnica de Madrid, Madrid (Spain) \\ \{elena.dominguez,gabriela.sansigre,josedavid.oses,david.luengo\}@upm.es
}

\begin{abstract}
During the last decades, power line communications (PLC) has emerged as an alternative to other wired line and wireless technologies. In particular, PLC has gained large acceptance for in-home scenarios, where it has become a competitive alternative to WiFi. Most PLC approaches are based on Multicarrier Modulation (MCM) using the Discrete Fourier Transform (DFT) as the basis to construct the multiple orthogonal channels. In this work, we explore the construction of MCM-PLC schemes based on the Discrete Cosine Transform Type-III even (DCT3e). We consider both SISO (single input single output) and MIMO (Multiple Input Multiple Output) PLC schemes, showing that they are a feasible alternative for in-home PLC applications.

Index Terms-Power Line Communications (PLC), Multicarrier Modulation (MCM), Discrete Cosine Transform (DCT), channel estimation.
\end{abstract}

\section{INTRODUCTION}

During the last decades, power line communications (PLC) has emerged as an alternative to other wired line and wireless technologies. In particular, PLC has gained large acceptance for in-home scenarios, where it has become a competitive alternative to WiFi. Most PLC approaches are based on Multicarrier Modulation (MCM) using the Discrete Fourier Transform (DFT) as the basis to construct the multiple orthogonal channels [1].

In this work, we explore the construction of MCM-PLC schemes based on the Discrete Cosine Transform TypeIII even (DCT3e). This interest is due to the fact that several works have shown that discrete cosine transforms (DCTs) can outperform the DFT in certain situations, e.g., in the presence of carrier frequency offset (CFO) or in cases where high-density primary modulations are used [2], [3], [4]. We consider both SISO (single input single output) and MIMO (Multiple Input Multiple Output) PLC schemes, showing that they are a feasible alternative for inhome PLC applications. MCM systems require a channel estimation stage, and afterwards, for any transmitted signal we need a reconstruction stage. For the channel estimation stage, several procedures have been proposed by using the

This work has been supported by the Spanish Ministry of Economy and Competitiveness through project TEC2015-64835-C3-3-R. Three of the authors are members of the TACA Research Group of Universidad Politécnica de Madrid (UPM). The first two authors are affiliated to ETSII (UPM), and the last ones to ETSIST (UPM). different DCTs [5]. In particular, some works have studied channel estimation via DCT3e [6], [7].

Regarding the reconstruction stage, in general, all MCM systems require inserting some kind of redundancy in the transmitted signal in order to avoid inter-symbol interference (ISI) and allow a perfect reconstruction (in the absence of noise in the receiver). In OFDM systems, which are based on the DFT, this redundancy typically consists in appending a cyclic prefix (CP) to the transmitted data, although some authors have proposed the use of zero padding (ZP) for DFT instead of the CP [8]. In DCTbased MCM schemes, [9] studied the kind of redundancy that had to be appended to the transmitted symbol in order to allow for perfect reconstruction at the receiver for the different types of DCTs. In [10], order to overcome this drawback, we proposed a new reconstruction scheme by using the DCT3e, showing that the whole reconstruction process can be performed exclusively by using the DCT3e.

The aim of this work is to apply the most efficient DCT3e techniques for both channel estimation and reconstruction, previously developed by the authors for SISO wireless communication systems, in the context of SISO/MIMO-PLC. As far as we know, this is the first time that a DCT-based MIMO-PLC scheme is proposed in the literature. The rest of the paper is organized as follows. Section II describes the channel estimation procedure by means of the DCT3e. Once the channel has been estimated, then Section III explains how to recover any transmitted signal using the DCT3e. Section IV applies the estimation and reconstruction procedures of the previous sections to the PLC problem. Finally, the conclusions are provided in Section $\mathrm{V}$.

\section{Channel Estimation VIA DCT3E-MIRAS}

We consider the general channel estimation problem of Fig. 1: our aim is to estimate the unknown $L$-length channel impulse response, $\mathbf{h}=\left[h_{0}, \cdots, h_{L-1}\right]^{T}$, by means of an $N$-length training symbol $\mathbf{x}$, which is known, and a discrete transform $\mathbf{T}$. The received signal is $\mathbf{y}=\mathbf{x} * \mathbf{h}+\mathbf{z}$, of length $L+N-1$, where $\mathbf{z}$ is a term related to the additive noise and $*$ denotes linear convolution. In the receiver, the aim is to estimate $\mathbf{h}$, by means of the 1-tap 


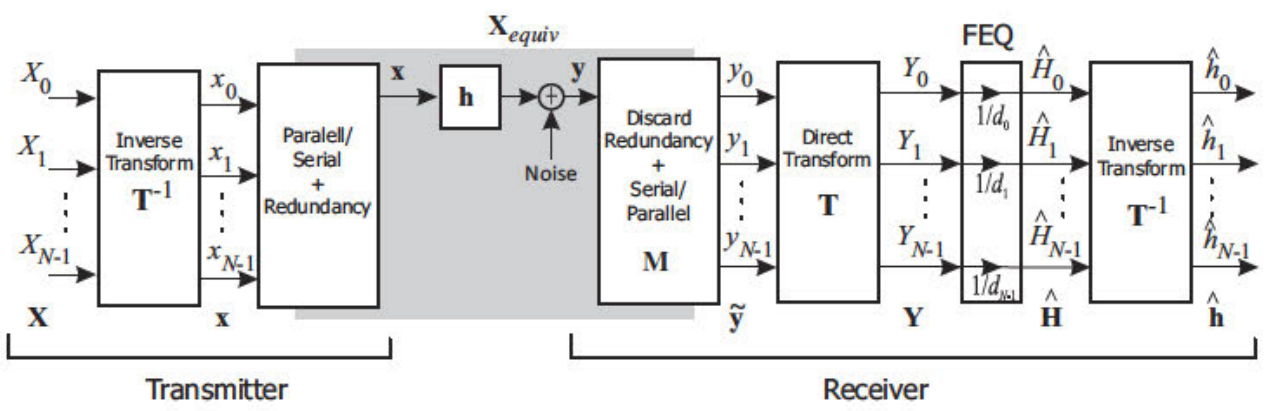

Fig. 1. General block diagram for channel estimation in MCM transceivers.

coefficients $d_{i}$, which are known a priori because they depend on the information in $\mathrm{x}$ and the transform $\mathrm{T}$.

In this work we make use of the DCT3e as the transform $\mathrm{T}$ both at the transmitter and the receiver. Recall that the DCT3e of an $N$-length signal is given by the matrix $\mathrm{C}_{3 e}$ whose entries are [11]

$$
\left(\mathbf{C}_{3 e}\right)_{k, j}=2 \alpha_{j} \cos \left(\frac{\pi(2 k+1) j}{2 N}\right) \quad k, j=0, \ldots, N-1
$$

with $\alpha_{0}=\alpha_{N-1}=1 / 2$, and $\alpha_{j}=1$ otherwise. We only need a known symbol with whole-sample (WS) symmetry:

$$
\mathbf{x}=\left[x_{M}, \cdots, x_{1}, x_{0}, x_{1}, \cdots, x_{M}\right]^{T},
$$

whose right-half part, zero-padded up to length $N$, is denoted as $\mathbf{x}_{Z P}^{r}=\left[x_{0}, x_{1}, \cdots, x_{M}, 0, \cdots, 0\right]^{T}$.

For our purpose, we need to define the auxiliary MIRAS procedure $\mathrm{M}$ that transforms an arbitrary vector of length $N+2 M=4 M+1, \mathbf{c}=\left[c_{0}, c_{1}, \ldots ., c_{4 M}\right]^{T}$, in the following way:

$$
\begin{aligned}
& \mathbf{M} \cdot \mathbf{c}=\left[c_{M}, . ., c_{2 M-1}, c_{2 M}, c_{2 M+1}, c_{2 M+2}, \ldots, c_{3 M}\right]^{T} \\
& +\left[c_{M}, \ldots, c_{1}, \quad 0, \quad 0,-c_{4 M}, . .,-c_{3 M+2}\right]^{T} .
\end{aligned}
$$

Notice that the final vector discards its first component, $c_{0}$, and produces a reversal (mirror symmetry) of the following $M$ components, adding them to the adjacent ones (replicating $c_{M}$ ); it also produces a mirror symmetry of its last $M-1$ components, and substracts them from the previous ones (discarding $c_{3 M+1}$ ). Therefore, it is a "mirror, replicate, add/subtract" procedure that we call MIRAS.

With these settings, we have proven [6] that the following procedure with DCT3-MIRAS provides an efficient channel estimation technique:

\section{SUMMARY OF THE CHANNEL ESTIMATION PROCEDURE:}

1) Choose a training signal $\mathrm{X}$ of odd length $N$, such that $\mathrm{x}=\mathrm{C}_{3 e}^{-1} \cdot \mathrm{X}$ is a WS signal.

2) Compute the DCT3e of the right-hand vector of $x$ : $\mathrm{C}_{3 e} \cdot \mathbf{x}_{Z P}^{r}=\mathbf{d}$, and store it in memory.
3) Transmit $x$ through the channel, obtaining the vector $\mathrm{y}$ at the receiver.

4) Append zeroes to the right of $y$ and modify it by the MIRAS procedure (mirror the edge components, replicate and add/substract them to/from their adjacent ones), in order to get the vector $\widetilde{\mathbf{y}}$ of length $N$, as depicted in Fig. 2:

- If $L \leq M+1$, simply apply MIRA ("mirror, replicate and add") only to the first $M$ components of $\mathbf{y}$, and then perform zero-padding to obtain an $N$-length vector $\widetilde{\mathbf{y}}$.

- If $L>M+1$, we apply MIRA to the first $M$ components of $\mathbf{y}$ and also apply "mirror and substract" to the $L-(M+1)$ final samples of $\mathbf{y}$, obtaining the $N$-length vector $\widetilde{\mathbf{y}}$.

5) Apply the $N$-point DCT3e block: $\mathrm{Y}=\mathrm{C}_{3 e} \cdot \widetilde{\mathbf{y}}$

6) Compute $\widehat{\mathbf{H}}_{k}=\mathbf{Y}_{k} / d_{k}$ by means of the 1-tap per subcarrier coefficient obtained in Step 2.

7) Obtain $\widehat{\mathbf{h}}_{z p}=\mathbf{C}_{3 e}^{-1} \cdot \widehat{\mathbf{H}}$ by an $N$-point inverse DCT3e, which is the desired estimation of the zero padded channel filter, i.e., $\widehat{\mathbf{h}}_{z p}=[0, \widehat{\mathbf{h}}, 0, \ldots, 0]$.

Notice that this procedure only makes use of the DCT3e transform, no extra transforms are needed, and it yields exact estimation in absence of noise. For this reasons, it will be used for the first time for PLC channel estimation, as explained in Section IV.

\section{SignAL ReCONSTRUCtion}

In this section we summarize how to perform the reconstruction of a transmitted signal by means of the DCT3e. The details and proofs can be found in [10].

In order to apply the diagonalization via DCT3e, the symmetry of the filter is needed. The estimated filter in the preceding section could not be symmetric, so the estimated filter is symmetrized by a convolution with a suitable prefilter. In the following

$$
\mathbf{h}=\left[h_{\nu}, \ldots, h_{1}, h_{0}, h_{1}, \ldots, h_{\nu}\right]
$$




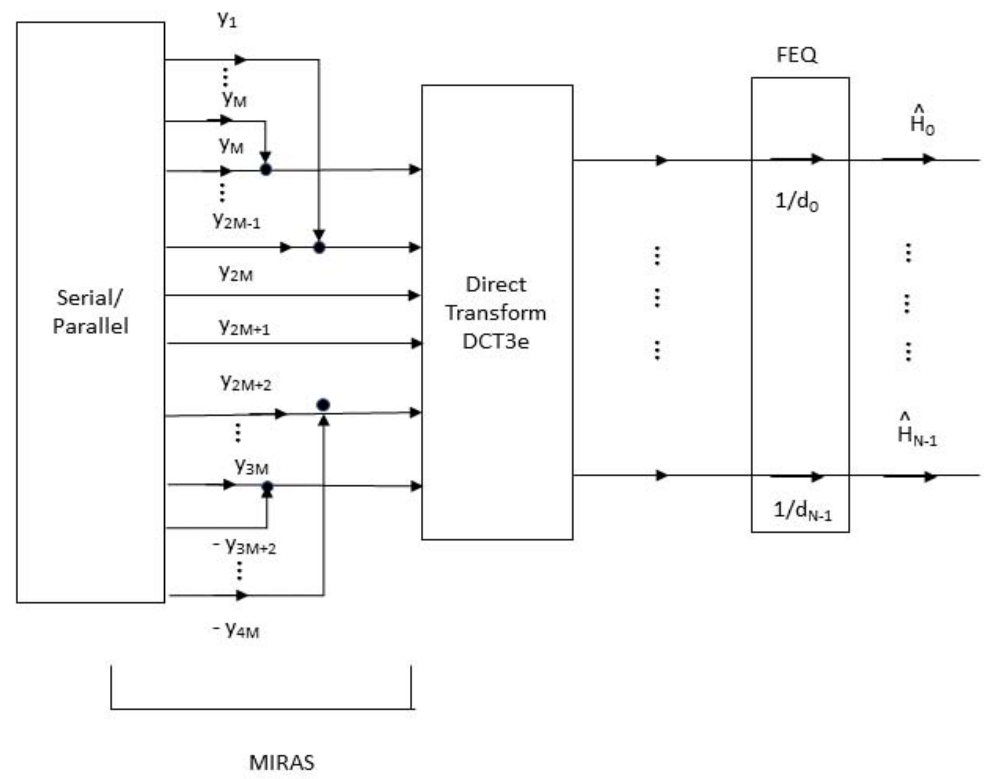

Fig. 2. MIRAS procedure at the receiver to compute $\widetilde{\mathbf{y}}$, followed by a DCT3e to obtain $\mathbf{Y}$, and division by $d_{k}$ to estimate the channel.

is a symmetric filter of length $2 \nu+1$. Its right-half part padded with zeros is

$$
\mathbf{h}^{r, z p}=\left[h_{0}, h_{1}, \ldots, h_{\nu}, 0, \ldots\right] .
$$

With these settings, we explain here how to reconstruct any transmitted signal by means of the DCT3e, being $\mathbf{h}$ the channel filter. This procedure also makes use of the MIRAS transformation (1).

\section{SUMMARY OF THE SIGNAL RECONSTRUC-} TION PROCEDURE:

1) For the symmetric filter $\mathbf{h}$ we store the coefficients $\mathbf{d}=\mathbf{C}_{3 e} \cdot \mathbf{h}^{r, z p}$ at the receiver.

2) Any original $N$-length vector $\left[X_{0}, \ldots, X_{N-1}\right]$, is extended to a $(N+1)$ - length vector $\mathbf{X}$ as

$$
\mathbf{X}=\left[X_{0}, \ldots, X_{N-1},-\sum_{j=0}^{N-1} X_{j}\right]^{T}
$$

3) Compute the $(N+1)$ point IDCT3e of the vector $\mathbf{X}$, so that $\mathbf{C}_{3 e}^{-1} \cdot \mathbf{X}=\mathbf{x}_{z p}$, where $\mathbf{x}_{z p}^{T}=\left[0, \mathbf{x}^{T}\right]$ has length $N+1$.

4) Transmit $\mathbf{x}_{z p}$ through the channel and receive the vector $\mathbf{y}$, which has $N+2 \nu+1$ components.

5) Apply the MIRAS procedure to the first $\nu$ and last $\nu-1$ components of $\mathbf{y}$ in order to get $\tilde{\mathbf{y}}$ of length $N+1$.

6) Compute $\mathbf{Y}=\mathbf{C}_{3 e} \cdot \tilde{\mathbf{y}}$ and obtain the reconstructed signal $\widehat{\mathbf{X}}_{k}=\mathbf{Y}_{k} / d_{k}$ for $k=0, \ldots, N-1$.

\section{NUMERICAL RESUlTS}

In order to evaluate the performance of the proposed SISO and MIMO systems, we test them using the model developed by researchers from Universidad de Málaga for in-home PLC communications [12]. This model is based on the theory of multiple transmission lines, and provides the impulse response (and the associated frequency response) for a given network topology. Figure 3 shows the channel's topology considered: several sections of cable with stubs loaded with different impedances between the generator (i.e., the transmitter) and the load $Z_{L}$ (i.e., the receiver). For the simulations, we have considered three cases with an increasing number of sections (5, 7 and 9). Their energy-normalized impulse and frequency responses are displayed in Figure 4. Notice the increasing length of the impulse response and the deeper spectral nulls as the number of sections increases, especially when we move from 5 to 7 sections.

For the simulations, we fix the number of subcarriers, $N=1023$, and perform $N_{s}=10^{5}$ experiments for each channel and SNRs ranging from $-10 \mathrm{~dB}$ to $50 \mathrm{~dB}$. We use the channel estimation approach described in Section II to estimate the channel, constructing a pre-filter $\widehat{\mathbf{w}}$ from the estimated channel's impulse response, $\widehat{\mathbf{h}}_{c h}$. Note that this will result in a total impulse response which will not be perfectly symmetric, with larger asymmetries for smaller SNR values, as the estimated impulse response will be worse in this case. Since the model used corresponds to a SISO channel, we extend it to the MIMO case following the simple approach suggested in [13]: the two direct channels are set using the same SISO model and the two 


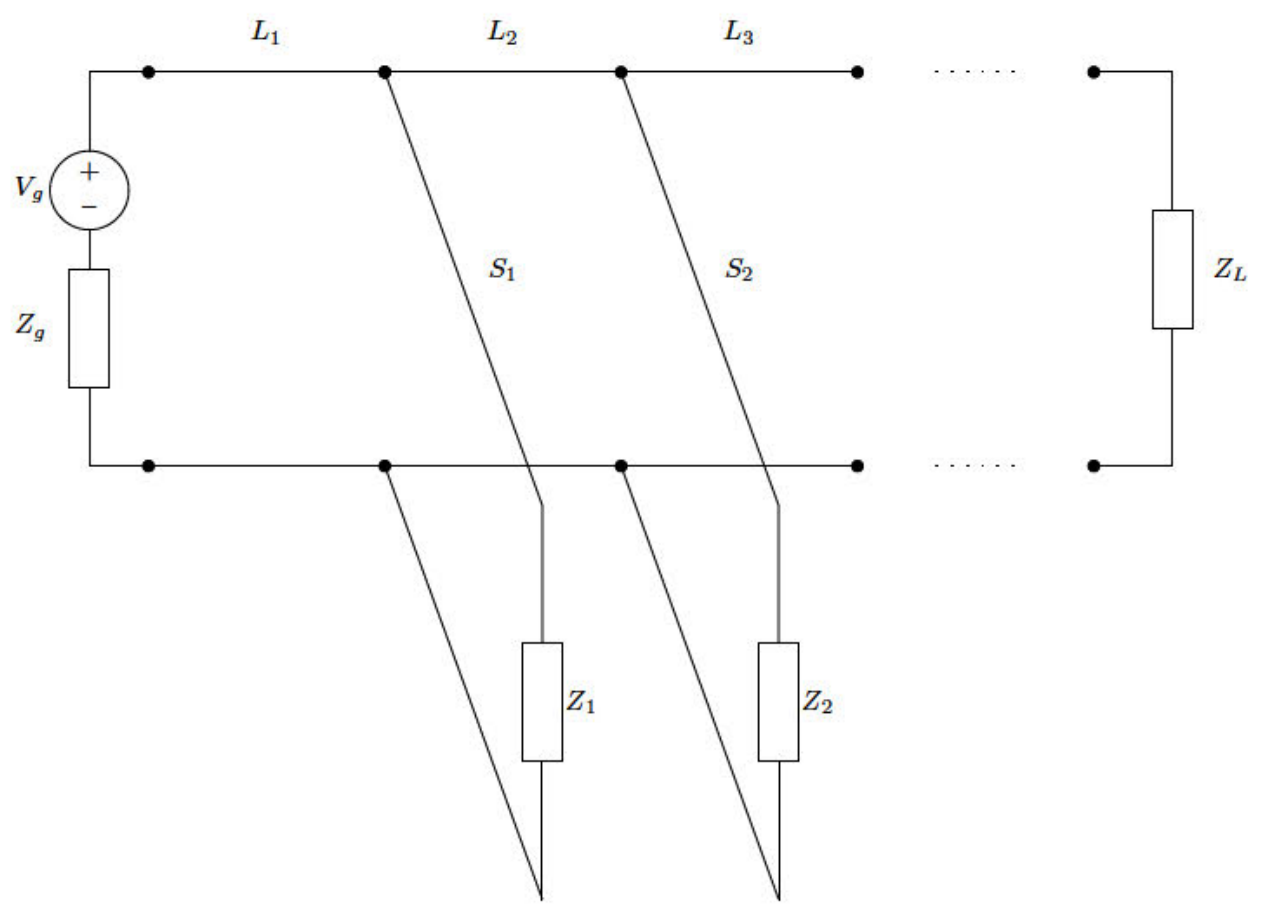

Fig. 3. Schematic representation of the electrical network topology considered: $V_{g}$ and $Z_{g}$ indicate the transmitter's voltage and impedance, respectively; $Z_{L}$ is the receiver's impedance; $L_{1}, L_{2}, L_{3}, \ldots$ are the lengths of the different sections; and $S_{1}, S_{2}, \ldots$ are the lengths of the stubs.
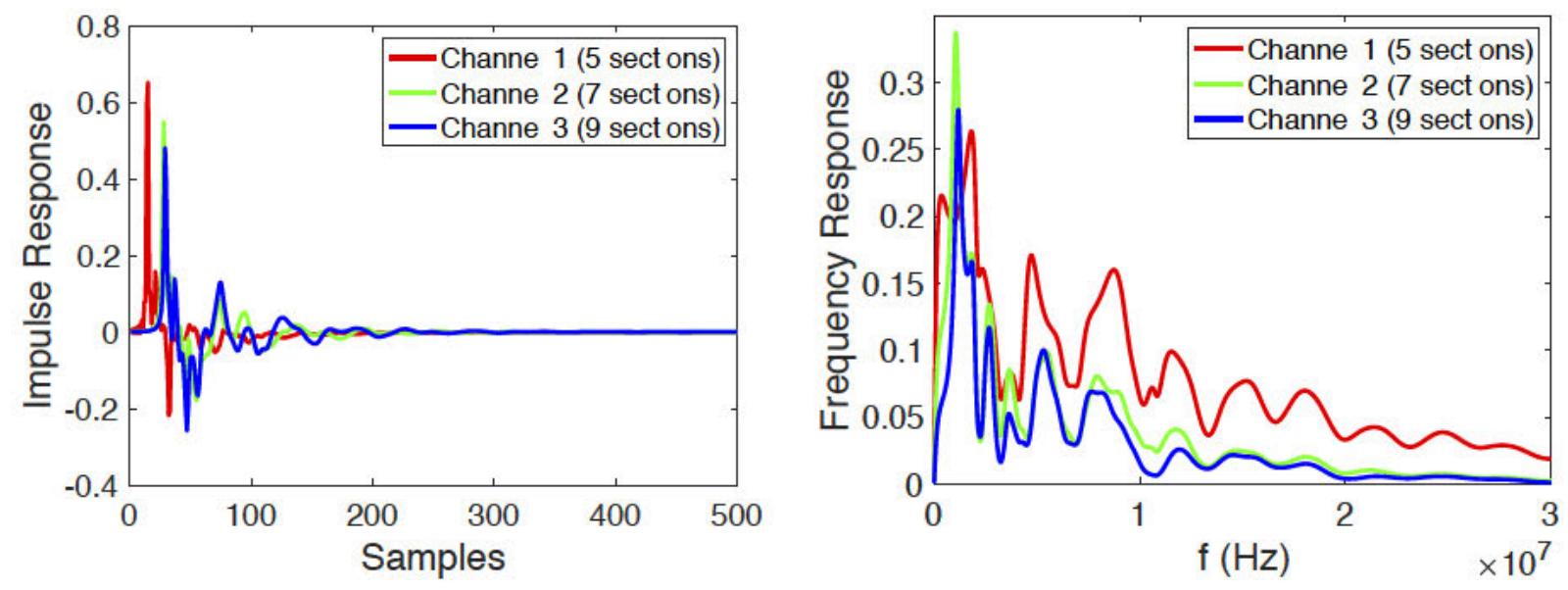

Fig. 4. Three considered channels: impulse response and frequency response.

cross channels are generated simply by considering an attenuation factor $\rho=0.9$. This approach is justified by the high correlation existing for in-home PLC communications, where the same path is typically followed by all the channels.

Figure 5 shows the bit error rate (BER) for the SISO and MIMO schemes using the three channels considered. On the one hand, note the increased BER for all the PLC channels w.r.t. the additive white Gaussian noise (AWGN) channel. This increased BER is due to the fact that PLC channels are much more challenging than the AWGN channel and no error control coding has been used.
In particular, note the large gap in performance between channel 1 (5 sections) and channels 2 and 3 (7 and 9 sections). On the other hand, note the slight increase in performance provided by the MIMO scheme, even though the optimal space-time coding approach has not been used. This moderate increase is due to the high correlation among channels, which implies a much lower degree of diversity than in wireless scenarios.

\section{Conclusions}

In this paper, we describe a simple method for channel estimation and signal reconstruction in multicarrier communications using the DCT3e. The proposed approach is 


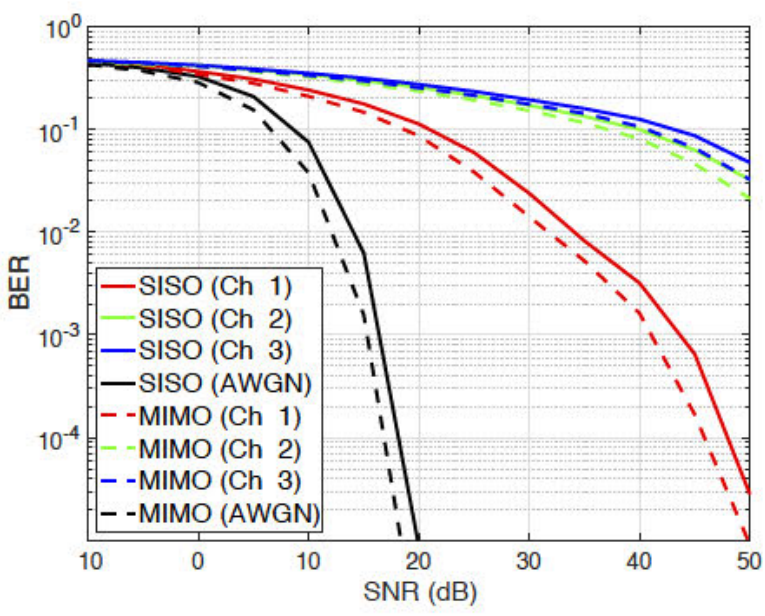

Fig. 5. Bit error rate (BER) for the SISO and MIMO schemes using the three channels considered.

then applied to realistic channels that can be found in indoor power line communications. Both a SISO and a MIMO communication scheme are considered. Numerical simulations show the good performance of the proposed approach for channel estimation and signal reconstruction.

\section{REFERENCES}

[1] C. Cano, A. Pittolo, D. Malone, L. Lampe, A. M. Tonello, and A. G. Dabak, "State of the art in power line communications: From the applications to the medium," IEEE Journal on Selected Areas in Communications, vol. 34, no. 7, 1935-1952, 2016.

[2] N. Al-Dhahir, "Optimum DCT-Based Multicarrier Transceivers for Frequency-Selective Channels", IEEE Trans. Commun., Vol. 53(5), pp. 911-921, May 2006.

[3] P. Tan, and N. C. Beaulieu, "A comparison of DCT-based OFDM and DFT-based OFDM in frequency offset and fading channels," IEEE Transactions on Communications, Vol. 54(11), pp. 21132125, Nov. 2006

[4] F. Cruz-Roldán, M. E. Domínguez-Jiménez, G. Sansigre-Vidal, J. Pineiro-Ave, and M. Blanco-Velasco, "Single-carrier and multicarrier transceivers based on discrete cosine transform type-IV," IEEE Transactions on Wireless Communications, vol. 12, no. 12, pp. 6454-6463, Dec. 2013.

[5] F. Cruz-Roldán, M. E. Domínguez-Jiménez, G. Sansigre-Vidal, D. Luengo, and M. Moonen, "DCT-based channel estimation for single- and multicarrier communications," Signal Processing., vol. 128, pp. 332-339, Nov. 2016.

[6] M. E. Domínguez-Jiménez, D. Luengo, G. Sansigre-Vidal "Channel Estimation Based on the Discrete Cosine Transform Type-III Even", Proceedings of the 26th European Signal Processing Conference (EUSIPCO 2018), pp. 1297-1301, Sept. 2018.

[7] M. E. Domínguez-Jiménez, D. Luengo, F. Cruz-Roldán, "Channel Estimation using Type-III even Discrete Cosine Transform in Multicarrier Communications", Proceedings of the IEEE Workshop on Statistical Signal Processing (SSP 2018), Friburg, June 2018.

[8] B. Muquet, Z. Wang, G. B. Giannakis, M. de Courville, and P. Duhamel, "Cyclic Prefixing or Zero Padding for Wireless Multicarrier Transmissions?", IEEE Trans. Commun., Vol. 50(2), pp. 2136-2137, Dec. 2002.

[9] F. Cruz-Roldán, M. E. Domínguez-Jiménez, G. Sansigre-Vidal, P. Amo-López, M. Blanco-Velasco, and Á. Bravo-Santos, "On the use of discrete cosine transforms for multicarrier communications," IEEE Transactions on Signal Processing, vol. 60, no. 11, pp. 60856090, Nov. 2012.
[10] M. E. Domínguez-Jiménez, G. Sansigre-Vidal, and D. Luengo, "Signal Reconstruction in Multicarrier Comunications by Means of the Discrete Cosine Transform Type-III Even," Proceedings of the 41st International Conference on Telecommunications and Signal Processing (TSP 2018), pp. 498-502, July 2018.

[11] S. A. Martucci, "Symmetric Convolution and the Discrete Sine and Cosine Transforms", IEEE Trans. Signal Processing, Vol. 32(5), pp. 1038-1051, May 1993.

[12] F. J. Cañete, J. A. Cortés, L. Díez, and J. T. Entrambasaguas, "A channel model proposal for indoor power line communications," IEEE Communications Magazine, vol. 49, no. 12, 166-174, 2011.

[13] J. A. Corchado, J. A. Cortés, F. J. Cañete, and L. Díez, "An MTL based channel model for indoor broadband MIMO power line communications." IEEE Journal on Selected Areas in Communications, vol. 34 , no. 7, 2045-2055, 2016. 